\title{
Specific Modification of an Arginine Residue in Mouse Contrapsin by Peptidylarginine Deiminase Altered Its Inhibitory Activities against Trypsin and Chymotrypsin ${ }^{\dagger}$
}

\author{
Hidenari TaKahara and Kiyoshi Sugawara \\ Laboratory of Biochemistry, Department of Agricultural Chemistry, \\ Ibaraki University, Ami-machi, Inashiki-gun, \\ Ibaraki 300-03, Japan \\ Received January 13, 1987
}

\begin{abstract}
We studied the effects of peptidylarginine deiminase, a $\mathrm{Ca}^{2+}$-dependent protein-modulating enzyme that catalyzes the deimination of arginyl residues in protein on two major trypsin inhibitors in mouse plasma. One of these inhibitors was $\alpha-1$-antitrypsin and the other was a recently characterized trypsin inhibitor termed contrapsin (H. Takahara and H. Sinohara, J. Biol. Chem., 257, 2438 (1982)). The enzyme abolished the trypsin-inhibiting activity of contrapsin in a process that was pseudo-first order with the rate dependent on enzyme concentration (second order rate constant $=3.4 \times 10^{3} \mathrm{M}^{-1} \cdot \mathrm{s}^{-1}$ ), but no detectable changes in the activity was noted for $\alpha-1$ antitrypsin. Millimolar $\mathrm{Ca}^{2+}$ and dithiothreitol were absolutely required for the inactivation of contrapsin by peptidylarginine deiminase. Although no significant alterations of the charge and the size in modified contrapsin were observed, the modified inhibitor indicated that 1 arginyl residue was converted to a citrullyl residue. Modified contrapsin whose anti-tryptic activity was lost inhibited chymotrypsin much more strongly than the native inhibitor. These data suggest that a vital amino acid residue for the anti-tryptic activity of mouse contrapsin is an arginyl residue and the conversion of this arginyl residue to a citrullyl residue causes the functional changes of the inhibitor.
\end{abstract}

Peptidylarginine deiminase (protein-arginine deiminase, protein L-arginine iminohydrolase, EC 3.5.3.15) catalyzes the modification of proteins through the conversion of arginine residues to citrulline residues. This enzyme activity was first found in a hair follicle extract ${ }^{1)}$ and was subsequently shown to distribute widely in tissues of vertebrates. ${ }^{2 \sim 7)}$ To date we have purified peptidylarginine deiminases from several tissues of animals ${ }^{7,8)}$ to electrophoretical homogeneity and reported the several properties of these enzymes including the substrate specificities. ${ }^{8}$ Especially, our recent findings that peptidylarginine deiminase from rabbit skeletal muscle selectively mod- ified the functional arginine residue of soybean trypsin inhibitor (Kunitz) (STI) ${ }^{9)}$ made this enzyme available for studies on the role of arginine residues in protein-proteinase inhibitors.

In this study we have examined the effects of peptidylarginine deiminase on two major trypsin inhibitors in mouse plasma. One of these inhibitors was $\alpha-1$-antitrypsin, whose molecular properties are very similar to those of homologous proteins isolated from several mammals. ${ }^{10)}$ The other is a newly identified inhibitor, tentatively termed contrapsin, which is lacking in human serum. ${ }^{10)}$ Although these two mouse trypsin inhibitors bear some re-

\footnotetext{
+ This work was supported in part by a Grant-in-Aid for Scientific Research (No. 60760062) from the Ministry of Education, Science and Culture of Japan.

Abbreviations: STI, soybean trypsin inhibitor (Kunitz); HEPES, 4-(2-hydroxyethyl)-1-piperazineethanesulfonic acid.
} 
semblance to each other with respect to chemical compositions, molecular weight, and helical compositions, they differ in antigenicity and the inhibitory spectra did not overlap each other with the exception of trypsin. ${ }^{10 \sim 13}$ ) Recently, Hill et al. ${ }^{14)}$ reported the sequences of cDNAs which represent the C-terminal halves of the two major murine plasma trypsin inhibitors. By comparison of the data with the known sequences for human plasma protease inhibitors, they showed that the sequence of mouse $\alpha$-1-antitrypsin is very similar to that of its human counterpart and contrapsin is highly homologous with the primate $\alpha-1$ antichymotrypsin. ${ }^{15)}$ Since the reactive center regions of human $\alpha$-1-antitrypsin ${ }^{16)}$ and $\alpha-1$ antichymotrypsin ${ }^{17)}$ are located towards the Cterminal of the proteins, they predicted the positions of the reactive center regions in these murine trypsin inhibitors by the alignment of the murine inhibitor sequences with the related human sequences. ${ }^{14)}$ However, the reactive sites designated $\mathrm{P}_{1}$ and $\mathrm{P}_{1^{\prime}}{ }^{18)}$ which are specifically cleaved by the target proteases are not clearly identified.

In this paper we described how a vital arginine residue in the reactive center of mouse contrapsin was preferentially modified by peptidylarginine deiminase and the predictable amino acids located at $\mathrm{P}_{1}$ and $\mathrm{P}_{1}$, were arginine and lysine, respectively. We also revealed that mouse contrapsin modified by peptidylarginine deiminase inhibited chymotrypsin much more strongly than the native inhibitor.

\section{MATERIALS AND METHODS}

Chemicals. Benzoyl-L-arginine- $p$-nitroanilide was obtained from the Peptide Institute Inc., Mino, Osaka. Succinyl-L-alanyl-L-alanyl-L-prolyl-L-phenylalanine- $p$ nitroanilide was a gift from Dr. Hiroshi Ogawa, Department of Hygiene, Kinki University School of Medicine. Concanavaline A-Sepharose was purchased from Pharmacia. Other chemicals were the best available commercially.

Inhibitors and enzymes. Murine contrapsin and $\alpha-1$ antitrypsin were prepared as described previously. ${ }^{10}$ ) These inhibitors was essentially homogeneous by polyac- rylamide gel electrophoresis. Peptidylarginine deiminase was purified from rabbit skeletal muscle by the method described previously. ${ }^{19)}$ The enzyme preparation used in this study had the specific activity of 590 units/mg of protein, and was electrophoretically homogenous. Porcine trypsin and bovine chymotrypsin were purchased from the Sigma Chem. Co.

Modification with peptidylarginine deiminase. A typical modification of the murine trypsin inhibitor with peptidylarginine deiminase was done in $100 \mathrm{~mm}$ HEPES-NaOH buffer, $\mathrm{pH} 7.2,10 \mathrm{mM} \mathrm{CaCl}_{2}, 10 \mathrm{~mm}$ dithiothreitol, $5 \mu \mathrm{M}$ trypsin inhibitor, and the enzyme. The modification was started by the addition of the enzyme and incubated at $37^{\circ} \mathrm{C}$. After the appropriate incubation period, the incubation was ended by the addition of $25 \mathrm{~mm} \mathrm{Na}$. EDTA, $\mathrm{pH}$ 7.0, and assayed for residual trypsin-inhibiting activity.

Assays of protease-inhibiting activity. Protease-inhibiting activities were measured by the decrease in proteolytic activities after incubation with the inhibitors at $25^{\circ} \mathrm{C}$. The trypsin activity was measured using benzoyl-Larginine- $p$-nitroanilide by the method of Eriksson. ${ }^{20)}$ The chymotrypsin activity was measured by the method of Birk $^{21)}$ using succinyl-L-alanyl-L-alanyl-L-prolyl-L-phenylalanine- $p$-nitroanilide. Active trypsin and chymotrypsin were measured by the titration methods using $p$-nitrophenyl- $p^{\prime}$-guanidinobenzoate ${ }^{22)}$ and 2-hydroxy-5-nitrotoluenesulfonic acid. ${ }^{23)}$

Preparation of modified contrapsin. Contrapsin modified with peptidylarginine deiminase was obtained from a scaled-up reaction mixture. After the complete abolishment of the trypsin-inhibiting activity of contrapsin was confirmed, ammonium sulfate was added to a scaled-up reaction mixture to give $80 \%$ saturation. The resulting precipitate collected by centrifugation was dissolved in a minimal volume of $20 \mathrm{~mm}$ acetate buffer ( $\mathrm{pH}$ 6.4) containing $150 \mathrm{~mm} \mathrm{NaCl}, 1 \mathrm{~mm} \mathrm{CaCl}, 1 \mathrm{~mm} \mathrm{MgCl}_{2}$, and $1 \mathrm{~mm}$ $\mathrm{MnCl}_{2}$, and was dialyzed against the buffer. The dialysate was put on a column $(1 \times 9 \mathrm{~cm})$ of concanavalin ASepharose equilibrated with the same buffer. Since contrapsin is a glycoproteic inhibitor, the modified inhibitor in the dialysate was retained to the affinity adsorbent and the peptidylarginine deiminase present was passed through the column. After the column was washed with the equilibration buffer, the modified inhibitor was eluted with the buffer containing $100 \mathrm{~mm}$ methyl $\alpha$-D-mannopyranoside. The fraction containing modified contrapsin was collected and dialyzed against $50 \mathrm{~mm}$ Tris- $\mathrm{HCl}$ buffer $(\mathrm{pH} 7.5)$ containing $150 \mathrm{~mm} \mathrm{NaCl}$.

Analytical electrophoresis. Polyacrylamide gel electrophoresis in the absence and presence of sodium dodecyl sulfate were done in slab gels with $9 \sim 11 \%$ acrylamide by the methods of Davis ${ }^{24)}$ and Laemmli, ${ }^{25)}$ respectively. 
Amino acid analysis. Proteins were hydrolyzed in $6 \mathrm{~N}$ $\mathrm{HCl}$ at $110^{\circ} \mathrm{C}$ for $24 \mathrm{hr}$, and the amino acid compositions of the acid hydrolysates were found with a Hitachi model 855 analyzer, using sodium citrate buffer. For the measurement of citrulline residues in proteins, the samples were hydrolyzed in $6 \mathrm{~N} \mathrm{HCl}$ at $110^{\circ} \mathrm{C}$ for $16 \mathrm{hr}$ and the hydrolysates were analyzed using lithium citrate buffer. ${ }^{26)}$

Estimation of protein. The absorption coefficients $\left(A_{1 \mathrm{~cm}}^{1 \%}\right.$ at $280 \mathrm{~nm}$ ) and molecular weights used for calculating protein concentrations were: mouse contrapsin, 4.8 and $55,000^{10)}$; mouse $\alpha$-1-antitrypsin, 4.6 and $53,000^{10)}$; peptidylarginine deiminase from rabbit skeletal muscle, 16.6 and $83,000 .^{8,9)}$

\section{RESULTS}

Effects of peptidylarginine deiminase on trypsininhibiting activities of mouse contrapsin and $\alpha$-1-antitrypsin

When mouse contrapsin or $\alpha$-1-antitrypsin was incubated at $37^{\circ} \mathrm{C}$ with peptidylarginine deiminase in a molar ratio of $12: 1$, the enzyme progressively diminished the trypsin-inhibiting activity of the contrapsin. As shown in Fig. 1, complete inactivation of mouse contrapsin was observed after $3 \sim 4 \mathrm{hr}$ incubation. In contrast, no great changes of the activitiy of $\alpha-1$ antitrypsin were observed. Figure 2 shows that the inactivation of contrapsin by peptidylarginine deiminase was dependent on the enzyme concentration and the incubation time. A semilogarithmic plot of the residual trypsininhibiting activity of the inhibitor on the enzyme treatment was linear with respect to time, indicating pseudo-first order kinetics. The double reciprocal plot of the pseudo-first order rate constant which was calculated from the half-lives of the inhibitor versus the concentration of the enzyme was linear, with the second order rate constant $\left(k_{2} / K\right)$ of the inactivation $^{27)}$ close to $3.36 \times 10^{3} \mathrm{M}^{-1} \cdot \mathrm{s}^{-1}$ (data not shown).

Effects of $\mathrm{Ca}^{2+}$ and dithiothreitol on the inactivation of mouse contrapsin by peptidylarginine deiminase

The dependence on $\mathrm{Ca}^{2+}$ and dithiothreitol of the inactivation of mouse contrapsin by peptidylarginine deiminase was examined by

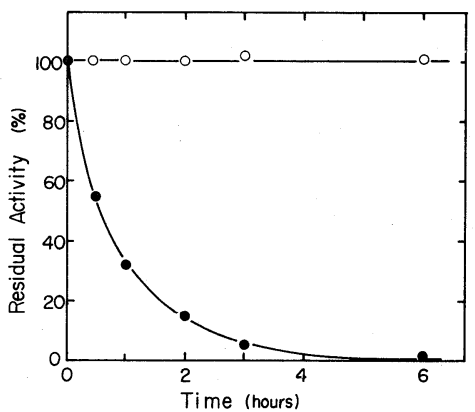

FIG. 1. Effects of Peptidylarginine Deiminase on the Trypsin-inhibiting Activities of Mouse Contrapsin and $\alpha$-1-Antitrypsin ( $\bigcirc)$.

The reaction mixture, which contained $100 \mathrm{~mm}$ HEPES$\mathrm{NaOH}$ buffer, pH 7.2, $10 \mathrm{~mm} \mathrm{CaCl}_{2}, 10 \mathrm{~mm}$ dithiothreitol, $5 \mu \mathrm{M}$ inhibitor, and $0.42 \mu \mathrm{M}$ peptidylarginine deiminase, in a total volume of $350 \mu \mathrm{l}$, was incubated at $37 \mathrm{C}$. At various times, the reaction was ended by adding $50 \mu \mathrm{l}$ of $200 \mathrm{~mm}$ $\mathrm{Na} \cdot$ EDTA, $\mathrm{pH} 7.0$, and then the mixture was assayed for the remaining trypsin-inhibiting activity as described in Materials AND Methods.

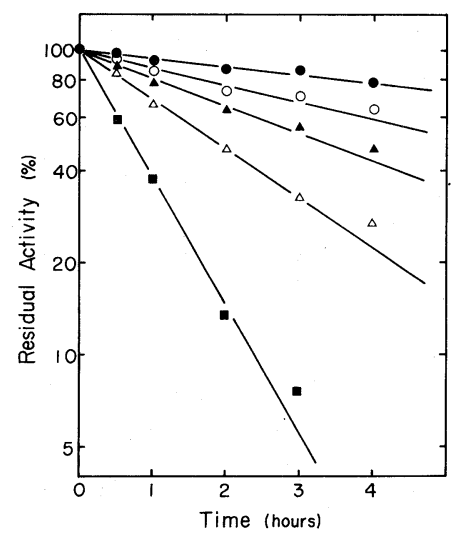

FIG. 2. Effects of Different Concentrations of Peptidylarginine Deiminase on the Trypsin-inhibiting Activity of Mouse Contrapsin.

Mouse contrapsin $(5.2 \mu \mathrm{M})$ was incubated at $37^{\circ} \mathrm{C}$ with different concentrations of peptidylarginine deiminase, $86 \mathrm{~nm}(\bigcirc), 108 \mathrm{~nm}(\bigcirc), 144 \mathrm{~nm}(\boldsymbol{\Delta}), 216 \mathrm{~nm}(\triangle), 432 \mathrm{~nm}$ $(\boldsymbol{\square})$, under the same conditions as in Fig. 1. At the indicated times, the reaction was ended by adding $50 \mu \mathrm{l}$ of $200 \mathrm{~mm} \mathrm{Na}$ EDTA, pH 7.0, and then the mixture was assayed for the remaining trypsin-inhibiting activity as described in Materials AND Methods.

omissions of the effectors from the reaction mixture. As shown in Fig. 3, the abolishment of the trypsin-inhibiting activity of contrapsin was not observed in the absence of $\mathrm{Ca}^{2+}$. 


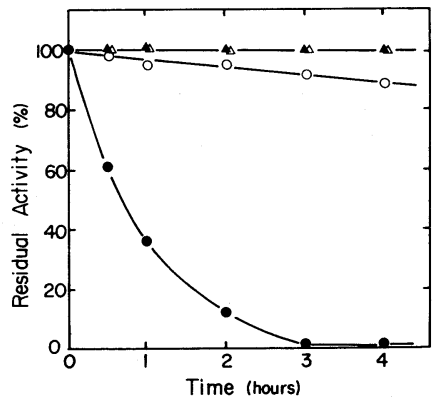

FIG. 3. Effects of $\mathrm{Ca}^{2+}$ and Dithiothreitol on the Modifying Activity of Peptidylarginine Deiminase towards Mouse Contrapsin.

Mouse contrapsin $(5 \mu \mathrm{M})$ was incubated with $0.42 \mu \mathrm{M}$ of peptidylarginine deiminase under the same conditions as in the legend to Fig. 1 (O), minus dithiothreitol (O), minus $\mathrm{Ca}^{2+}(\boldsymbol{\Delta})$, and minus both dithiothreitol and $\mathrm{Ca}^{2+}$ $(\triangle)$.

Furthermore, dithiothreitol was also almost essential for the reaction. The $\mathrm{Ca}^{2+}$ and dithiothreitol required for the half-maximal inactivation of contrapsin by the enzyme was about $0.4 \mathrm{~mm}$ and $1 \mathrm{~mm}$, respectively (data not shown).

\section{Polyacrylamide gel electrophoretic analysis of} the modification of mouse contrapsin by peptidylarginine deiminase

Changes in the charge and the size of the inhibitor at various stages of inactivation by the enzyme were assayed by polyacrylamide gel electrophoresis under non-denaturing ${ }^{24)}$ and denaturing conditions with sodium dodecyl sulfate. ${ }^{25)}$ As shown in Figs. 4A and 4B, the migration of contrapsin modified by peptidylarginine deiminase was not distinguished from that of the unmodified inhibitor in the absence and presence of sodium dodecyl sulfate. These suggest that the modification of contrapsin does not lead to significant changes in the net charge or the size of the molecule.

\section{Amino acid compositions of native and modified} contrapsin

For comparison of amino acid compositions in native and modified contrapsin, we prepared modified contrapsin from a scaled-up
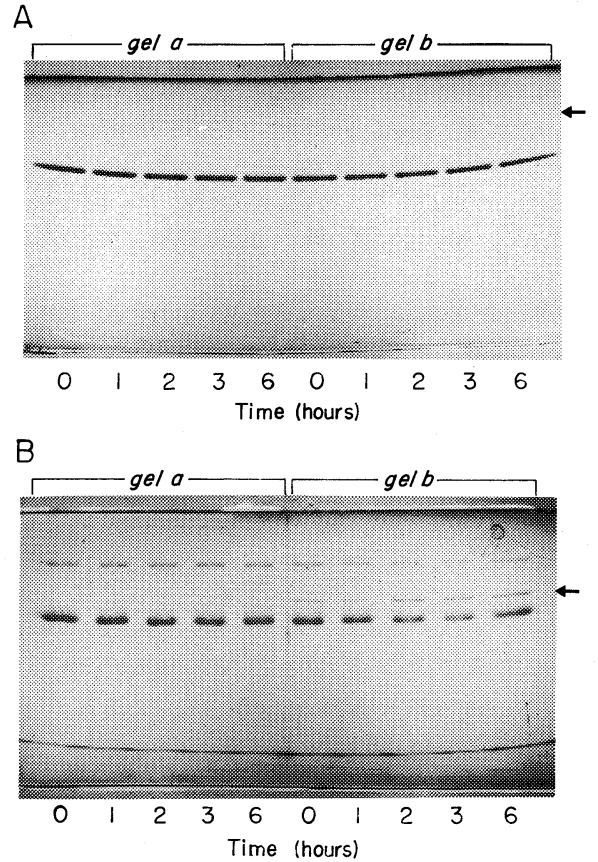

FIG. 4. Polyacrylamide Gel Electrophoretic Analysis of Inactivation of Mouse Contrapsin by Peptidylarginine Deiminase.

Mouse contrapsin $(5 \mu \mathrm{M})$ was incubated with $0.42 \mu \mathrm{M}$ of peptidylarginine deiminase under the same conditions as in the legend to Fig. 1. At the indicated times, the reaction was ended by adding $50 \mu \mathrm{l}$ of $200 \mathrm{~mm} \mathrm{Na} \cdot$ EDTA, pH 7.0, and then the reaction mixture $(10 \mu \mathrm{l})$ was used for polyacrylamide gel electrophoresis. A, polyacrylamide slab gel electrophoresis as described by Davis ${ }^{24)}$ using $11 \%$ acrylamide, stained with Coomassie Brilliant Blue. B, sodium dodecyl sulfate-polyacrylamide slab gel electrophoresis as described by Laemmli $^{25}$ ) using $9 \%$ acrylamide, stained with Coomassie Brilliant Blue. Gel electrophoretic patterns of mouse contrapsin with peptidylarginine deiminase ( $g e l b$ ) and without peptidylarginine deiminase ( $\mathrm{gel} a$ ). The protein band shown by the arrow $(\mathrm{gel} b$ ) indicates that with the enzyme added.

reaction mixture as described in MATERIALS AND METHODS. Except for the conversion of 1 arginyl residue to a citrullyl residue in modified contrapsin, no significant changes were noted in the contents of other amino acids (Table I). This indicates that 1 arginine residue essential for trypsin inhibitory activity in the inhibitor was selectively modified by peptidylarginine deiminase. 
Table I. Amino Acid Compositions of Native AND MOdified CONTRAPsin

Samples for amino acid analysis were prepared as described in Materials and Methods. The values were normalized to alanine and represent on the basis of 26 alanine residues/mol of contrapsin. ${ }^{10}$ )

\begin{tabular}{lcc}
\hline Amino acid & $\begin{array}{c}\text { Native } \\
\text { contrapsin }\end{array}$ & $\begin{array}{c}\text { Modified } \\
\text { contrapsin }\end{array}$ \\
\cline { 2 - 3 } & mol residues/mol inhibitor \\
\hline Aspartic acid & 39.5 & 39.6 \\
Threonine & 24.4 & 24.1 \\
Serine & 21.8 & 22.6 \\
Glutamic acid & 50.5 & 50.3 \\
Proline & 16.8 & 17.1 \\
Glycine & 14.2 & 13.5 \\
Alanine & 26.0 & 26.0 \\
Valine & 23.2 & 23.3 \\
Methionine & 9.7 & 9.7 \\
Isoleucine & 20.5 & 20.2 \\
Leucine & 41.0 & 40.8 \\
Tyrosine & 7.3 & 7.9 \\
Phenylalanine & 19.9 & 20.1 \\
Lysine & 26.5 & 26.1 \\
Histidine & 5.8 & 5.3 \\
Arginine & 9.3 & 8.0 \\
Citrulline & 0 & 1.0 \\
\hline
\end{tabular}

\section{Comparison of inhibition by native and modified contrapsin}

Inhibition by native and modified contrapsin against two pancreatic proteases were compared. Figure 5 shows that an inverse linear relationship exists between the amount of native contrapsin added and the remaining tryp$\sin$ activity. The extrapolated value of the concentration of the inhibitor to $100 \%$ inhibition is close to the amount of trypsin used in this experiment, suggesting that the native inhibitor formed an equimolar complex with trypsin. This agreed with our previous findings. ${ }^{10)}$ On the other hand, virtually no inhibition was observed when trypsin (0.93 nmol) was incubated with modified contrapsin $(1.5 \mathrm{nmol})$. In contrast to these data, modified contrapsin showed a much higher degree of inhibition against chymotrypsin than the native inhibitor (Fig. 6). Although the degree of the inhibition was linear with the amount of the modified inhibitor, the inhib-

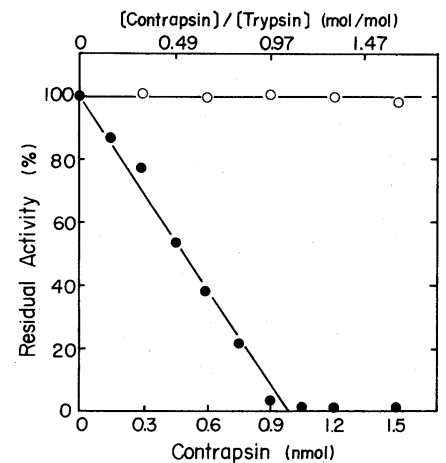

FIG. 5. Inhibition of Trypsin Activity as a Function of Native (O) and Modified ( $\bigcirc$ ) Contrapsin Concentration.

Various amounts of the inhibitors were preincubated with $0.93 \mathrm{nmol}$ of active porcine trypsin for $15 \mathrm{~min}$ at $25^{\circ} \mathrm{C}$, and the residual trypsin activities were measured as described in Materials and Methods.

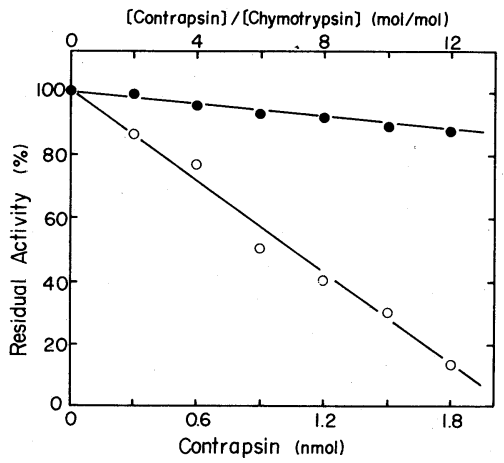

FIG. 6. Inhibition of Chymotrypsin as a Function of Native (O) and Modified ( $\bigcirc$ ) Contrapsin Concentration.

Various amounts of the inhibitors were preincubated with $0.5 \mathrm{nmol}$ of active bovine chymotrypsin for $15 \mathrm{~min}$ at $25 \mathrm{C}$, and the residual chymotrypsin activities were measured as described in Materials and Methods.

itory activity was not stoichiometric. The molecular mechanism underlying this phenomenon is not clear at present. It is possible that a substantial portion of modified contrapsin is instantaneously inactivated, while the remaining portion forms a stable complex with chymotrypsin.

\section{DISCUSSION}

The plasma protease inhibitors control a wide variety of physiological functions including blood coagulation, fibrinolysis, comple- 
ment activation, and inflammatory response. Contrapsin and $\alpha$-1-antitrypsin are major components of the protease inhibitory activities in mouse plasma. ${ }^{10,28)}$ In this study, treatment with peptidylarginine deiminase did not alter the inhibitory activity of mouse $\alpha-1$ antitrypsin (Fig. 1). Recently, Hill et al. ${ }^{14)}$ reported the sequences of cDNA and amino acids of mouse $\alpha$-1-antitrypsin and found no arginine residue in the reactive center region. Therefore, our results seem reasonable. On the other hand, peptidylarginine deiminase modified the functional arginine residue in contrapsin and abolished its inhibition of trypsin. However, the rate of the inactivation of contrapsin by the enzyme was much slower than that of the inactivation of STI as shown in our previous paper.9) The enzyme completely inactivated STI within $10 \mathrm{~min}$ using the enzyme to the inhibitor molar ratio of $1: 50$ at $37^{\circ} \mathrm{C} .^{9)}$ In the case of contrapsin, the inactivation was completed with $3 \sim 4 \mathrm{hr}$ under the same conditions (Fig. 1). These data suggest that the affinity of peptidylarginine deiminase for the functional arginine residue in contrap$\sin$ is less than that for its counterpart in STI. This view is supported by the kinetic data that the value of the second order rate constant for the inactivation of contrapsin by the enzyme, $3.36 \times 10^{3} \mathrm{M}^{-1} \cdot \mathrm{s}^{-1}$, was about one-tenth of that for the inactivation of STI. ${ }^{9 \text { ) }}$ Although the reason for this difference is unclear, it is presumably due to differences in the neighboring residues at the reactive site or to hindrance at the other sites.

Our preceding paper described how peptidylarginine deiminase required calcium ion as an essential cofactor and was strongly dependent on reducing reagents, such as dithiothreitol. ${ }^{8)}$ These properties were confirmed by this study showing the absolute requirements for calcium ion and dithiothreitol for the modification of contrapsin (Fig. 3). Recently, we have demonstrated that the role of $\mathrm{Ca}^{2+}$ in the expression of peptidylarginine deiminase activity should be to change its conformation from the inactive form to the active form. ${ }^{29)}$ On the other hand, the mech-

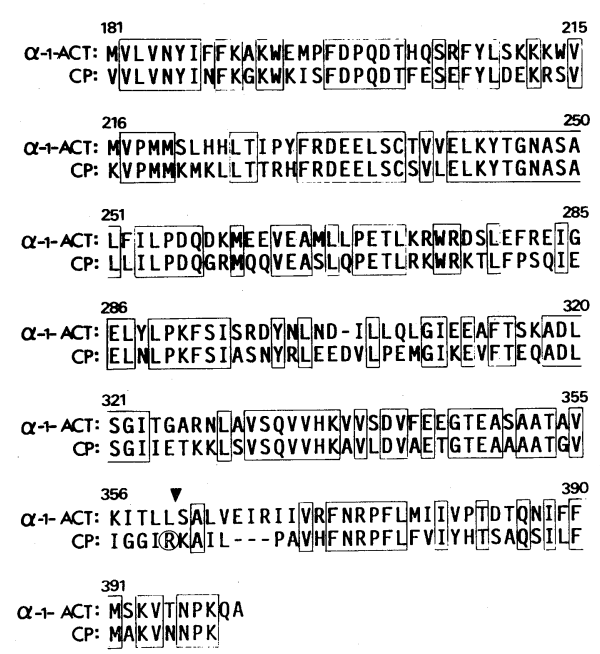

FIG. 7. Comparison of the Amino Acid Sequence of Mouse Contrapsin (CP) with That of Human $\alpha-1$ Antichymotrypsin $(\alpha-1-\mathrm{ACT})$.

Human $\alpha$-1-antichymotrypsin and mouse contrapsin sequences were obtained from Hill et al. ${ }^{14)}$ Amino acids are designated by the one-letter code. Numbers refer to the position relative to the $\mathrm{N}$-terminal asparagine residue of human $\alpha$-1-antichymotrypsin. ${ }^{31)}$ The sequences were aligned with gaps placed in regions of additions or deletions of amino acids. Residues which are identical between mouse contrapsin and human $\alpha$-1-antichymotrypsin are boxed. The arrowhead indicates the $\mathrm{P}_{1}-\mathrm{P}_{1}$, bond of human $\alpha$-1-antichymotrypsin which is cleaved during complex formation with bovine chymotrypsin. ${ }^{17)}$ The arginine residue enclosed with the circle is the putative amino acid residue modified by peptidylarginine deiminase.

anism of activation by dithiothreitol is not understood. Since mouse contrapsin appeared to have only one cysteinyl residue, this rules out the possibility that the strong dependency of the reaction on the reducing reagent is attributed to a chemical denaturation of the inhibitor by the reduction of disulfide groups. The reducing reagents may be necessary for the maintenance of the enzymatic activity during the prolonged incubation. As shown in our previous paper, the deimination of one arginyl residue in STI decreased the positive charge, and modified STI was distinguished from the native inhibitor by polyacrylamide gel electrophoresis under nondenaturing conditions. ${ }^{9)}$ In the case of contrapsin, the decrement of one arginyl residue by the enzymatic 
reaction did not affect the net charge in the inhibitor (Fig. 4), indicating that the arginine residue modified by the enzyme makes little contribution to the charge.

Recently, mouse contrapsin has been described as a "serpin", a superfamily of serine protease inhibitors which includes the human serine protease inhibitors $\alpha$-1-antitrypsin, antithrombin III, $\alpha$-1-antichymotrypsin, C1inhibitor, and $\alpha$-2-antiplasmin. ${ }^{30)}$ As shown in Fig. 7 which represents amino acid sequences of C-terminal halves of the inhibitors, mouse contrapsin is highly homologous with human $\alpha$-1-antichymotrypsin, an inhibitor of chymotrypsin-like proteases. In contrast with the similarity of the sequences, mouse contrapsin and human $\alpha$-1-antichymotrypsin differ greatly in their protease-inhibiting spectra. ${ }^{10 \sim 12)}$ Consistent with this, the reactive center regions of these sequences differ substantially. Since the $P_{1}$ residue of the reactive site is recognized by the target protease, an arginine or a lysine residue in the reactive center region of mouse contrapsin is a candidate for the $\mathrm{P}_{1}$ residue. This work, therefore, implies that $P_{1}$ residue of mouse contrapsin is the arginine residue which is enclosed within the circle as shown in Fig. 7. The functional significance of this arginine residue is of considerable interest because modified contrapsin shows a much higher degree of inhibition against chymotrypsin than the native inhibitor (Fig. 6). The results are consistent with the proposition of Hill et al. ${ }^{14)}$ in which mouse contrapsin is closely related to human $\alpha-1$ antichymotrypsin but has different inhibitory activity because of divergence within this functionally important stretch of amino acid residues.

Acknowledgments. We wish thank to Professor Hyogo Sinohara, Department of Biochemistry, Kinki University School of Medicine, for his valuable suggestions. The gift of chromogenic peptide substrate from Dr. Hiroshi Ogawa, Kinki University, is also gratefully acknowledged.

\section{REFERENCES}

1) G. E. Rogers and L. D. Taylor, "Advances in Experimental Medicine and Biology," Vol. 86A, ed. by M. Friedman, Plenum Press, New York and London, 1977, pp. 283 294.

2) K. Sugawara and M. Fujisaki, Agric. Biol. Chem., 43, 2407 (1979).

3) J. Kubilus, R. F. Waitkus and H. P. Baden, Biochim. Biophys. Acta, 615, 246 (1980).

4) K. Hosokawa, H. Takahara and K. Sugawara, Agric. Biol. Chem., 47, 1695 (1983).

5) J. Kubilus and H. P. Baden, Biochim. Biophys. Acta, 745, 285 (1983).

6) J. Kubilus and H. P. Baden, J. Invest. Dermatol., 85, 232 (1985).

7) H. Takahara, K. Sueyoshi and K. Sugawara, Agric. Biol. Chem., 50, 1303 (1986).

8) H. Takahara, Y. Oikawa and K. Sugawara, J. Biochem., 94, 1.945 (1983).

9) H. Takahara, H. Okamoto and K. Sugawara, J. Biol. Chem., 260, 8378 (1985).

10) H. Takahara and H. Sinohara, J. Biol. Chem., 257, 2438 (1982).

11) H. Takahara and H. Sinohara, Thromb. Res., 27, 45 (1982).

12) H. Takahara and H. Sinohara, J. Biochem., 93, 1411 (1983).

13) H. Takahara, S. Sibata and H. Sinohara, Tohoku J. Exp. Med., 142, 261 (1984).

14) R. E. Hill, P. H. Shaw, P. A. Boyd, H. Baumann and N. D. Hastie, Nature, 311, 175 (1984).

15) J. Travis, D. Garner and J. Bowen, Biochemistry, 17, 5647 (1978).

16) D. R. Boswell, J. O. Jeppsson, S. O. Brennan and R. W. Carrell, Biochim. Biophys. Acta, 744, 212 (1983).

17) M. Morii and J. Travis, J. Biol. Chem., 258, 12749 (1983).

18) M. Laskowski, Jr. and I. Kato, Annu. Rev. Biochem., 49, 593 (1980).

19) H. Takahara, H. Okamoto and K. Sugawara, J. Biochem., 99, 1417 (1986).

20) S. Eriksson, Acta Med. Scand., 177, Suppl. 432, 1 (1965).

21) Y. Birk, "Methods in Enzymology," Vol. 45, ed. by S. P. Colowick and N. O. Kaplan, Academic Press Inc., New York, 1976, pp. 716 722 .

22) T. Chase, Jr. and E. Shaw, Biochem. Biophys. Res. Commun., 29, 508 (1967).

23) F. J. Kezdy and E. T. Kaiser, "Methods in Enzymology," Vol. 19, ed. by S. P. Colowick and N. O. Kaplan, Academic Press Inc., New York, 1970, pp. $3 \sim 20$.

24) B. J. Davis, Ann. N. Y. Acad. Sci., 121, 404 (1964).

25) U. K. Laemmli, Nature, 227, 680 (1970).

26) K. Sugawara, Y. Oikawa and T. Ouchi, J. Biochem., 91, 1065 (1982).

27) R. Kitz and I. B. Wilson, J. Biol. Chem., 237, 3245 (1962).

28) H. Takahara, Y. Nakamura, K. Yamamoto and H. Sinohara, Tohoku J. Exp. Med., 139, 265 (1983). 
29) H. Takahara, H. Okamoto and K. Sugawara, Agric. Biol. Chem., 50, 2899 (1986).

30) R. Carrell, Nature, 312, 14 (1984).

31) S. C. Bock, K. Skriver, E. Nielsen, H. C. Thøgersen,
B. Wiman, V. H. Donaldson, R. L. Eddy, J. Marrinan, E. Radziejewska, R. Huber, T. B. Shows and S. Magnusson, Biochemistry, 25, 4292 (1986). 